\title{
Expressed Sequence Tag
}

National Cancer Institute

\section{Source}

National Cancer Institute. Expressed Sequence Tag. NCI Thesaurus. Code C18155.

Refers to Sequence Tagged Sites (STS) that are represented in mRNA. They are derived as 300-500 bp sing le-pass mRNA sequence reads from many of the genes expressed in a given tissue and/or at a given developmental stage. They represent a snapshot of gene expression in a given place or time. 